\title{
Paternal inheritance of mitochondrial DNA in Chlamydomonas reinhardtii and dynamic morphological changes in mitochondria, mitochondrial nucleoids, and the cell nucleus during its life cycle
}

\author{
Soichi Nakamura \\ Laboratory of Cell and Functional Biology, Faculty of Science, University of the Ryukyus, Nishihara, Okinawa 903-0213 \\ Author for correspondence: S. Nakamura, nsoichi@sci.u-ryukyu.ac.jp
}

\begin{abstract}
Summary: To determine the exact mode of inheritance of mitochondrial DNA (mtDNA) in Chlamydomonas reinhardtii, suitable markers to determine the origin of parental mitochondrial genes are necessary. For this goal, the difference in mtDNA between a $C$. reinhardtii strain and a $C$. smithii strain was used. These two species were serially backcrossed to ensure matching of the background of their nuclear genomes. The results showed strict paternal inheritance of mtDNA. Next, the fate of $\mathrm{mt}^{+}$(female) and mt (male) mtDNA was followed using three methods: quantitative real-time PCR, Southern blotting hybridization, and fluorescence in situ hybridization (FISH). The disappearance of $\mathrm{mt}^{+} \mathrm{mtDNA}$ was observed at the beginning of meiosis. In the mutant $b p 31$, in which the maternal inheritance of chloroplast DNA (cpDNA) is disrupted, the paternal inheritance of mtDNA was also found to be disrupted, indicating commonality between cpDNA and mtDNA in part of the process leading to uniparental inheritance. Mitochondrial nucleoids and mitochondria were observed in living vegetative cells. Organellar nucleoids were stained with SYBR Green I, while mitochondria were stained with $\mathrm{DiOC}_{6}$. During the cell cycle, mitochondrial nucleoids in living cells were granular, and mitochondria formed tangled threads. When mature zygotes were exposed to the light, meiosis began. Living zygotes with thick walls was also stained by SYBR Green I and $\mathrm{DiOC}_{6}$. At the diakinesis stage of meiosis, the total number of chromosomes was 18. When mature zygotes were exposed to the light, granular mitochondria began to form short thread-like structures and then assembled around the cell nucleus. Once assembled, the mitochondria began to scatter in the opposite hemisphere, forming long and tangled thread-like structures. Mitochondrial nucleoids were usually granular, but some of them developed bead-like structures, probably by dividing. The results from the molecular biochemical experiments suggested that $\mathrm{mt}^{+}$mitochondrial nucleoids disappear at the stage of assembly and the scattering of mitochondria. Then, the first and second nuclear divisions proceed, producing tetrads.
\end{abstract}

Key words: Chlamydomonas reinhardtii, $\mathrm{DiOC}_{6}$, mitochondrial DNA, paternal inheritance, SYBR Green I

\section{INTRODUCTION}

Mendel's laws were established in 1866 and non-Mendelian inheritance was discovered in 1909 by Correns and Baur. Correns (1909) followed the inheritance of green, variegated, and white patterns of leaves in reciprocal crosses of Mirabilis jalapa. He found that the color patterns are passed on to the next generation from only the maternal parent. In the same year, Baur (1909) found that the green and variegated patterns of the leaves in Pelargonium are inherited biparentally. Since then, studies of cytoplasmic organelle inheritance have established that most eukaryotes inherit chloroplasts and mitochondria maternally (Tilney-Bassett 1978, Kuroiwa 1991, 2010, Gillham 1994).

The uniparental inheritance of mitochondria and chloroplasts has been explained by the difference in size between female and male gametes as female gametes contain a large number of organelles and male gametes contain only a few (Gyllensten et al. 1991). However, the process leading to this inheritance is not simple because uniparental inheritance occurs even in isogamous unicellular green algae, Chlamydomonas reinhardtii (Sager 1954). Sager isolated the UV-induced mutant $s r 2$ that is resistant to streptomycin. When the $s r 2$ strain and wild-type cells were crossed in various ways, resistance in the meiotic progeny was inherited from the maternal parent (maternal inheritance). The progeny also inherited chloroplast genes from the $\mathrm{mt}^{-}$parent at a frequency of $1-10 \%$ (paternal inheritance). Biparental inheritance, involving the inheritance of both parental genes, also occurred at a low frequency. These maternal, biparental, and paternal types of inheritance involve inheritance that is non-Mendelian (Gillham 1994). Factors that undergo Mendelian inheritance are located on the chromosomes (Sutton 1902), while those that undergo non-Mendelian inheritance are thought to be located in the cytoplasm. Ris and Plaut (1962) revealed the presence of DNA in the chloroplasts by microscopy. This chloroplast DNA (cpDNA) was first isolated from C. reinhardtii cells by Sager and Ishida (1963) by centrifugation using $\mathrm{CsCl}$. Liu et al. (1989) subsequently identified the $s r 2$ mutation in the chloroplast genome.

C. reinhardtii has chloroplasts and mitochondria. The chloroplast nucleoids contain cpDNA, which is organized into 5-10 DNA-protein complexes (Kuroiwa et al. 1981, 1982). The mitochondrial DNA (mtDNA) is a $15.8 \mathrm{-kbp}$ linear molecule (Grant and Chiang 1980, Ryan et al. 1978). mtDNA is organized into mitochondrial nucleoids, of which approximately 20 are contained within a cell (Nishimura et al. 1998). Via the process of fertilization, $\mathrm{mt}^{+}$(female) and $\mathrm{mt}^{-}$(male) gametes form diploid zygotes. After the incubation of these zygotes in the dark for about one week, meiosis begins after exposure to light and four haploid daughter cells are usually produced (Sager 1954).

Sager and Lane (1972) reported the results of a $\mathrm{CsCl}$ centrifugation experiment using $C$. reinhardtii. Only male 
cpDNA disappeared in zygotes 6-24 h after zygote formation. Kuroiwa et al. (1982) stained cells with the DNA-specific fluorochrome 4',6-diamidino-2-phenylindole (DAPI) and presented the first cytological evidence that male chloroplast nucleoids disappeared completely during the first 40-50 min after fusion. Kuroiwa (1985) subsequently proposed an active digestion hypothesis to explain this uniparental inheritance. This hypothesis has been applied to both mitochondrial and chloroplast inheritance in plants (Kuroiwa 2010).

\section{Reported research on the inheritance of mtDNA in Chlamydomonas}

Boynton et al. (1987) performed interspecific crosses of $C$. smithii $\mathrm{mt}^{+}$and $C$. reinhardtii $\mathrm{mt}^{-}$strains and analyzed the inheritance pattern in progeny using the physical differences in restriction fragment patterns of mtDNA. All of the meiotic progeny showed paternal inheritance.

Matagne et al. (1989) used acriflavin as a mutagen and isolated two respiration-defective mutants, dum-1 and dum-2, having a $1.5-\mathrm{kbp}$ deletion in the mitochondrial cob gene. They grew in the light, but did not divide under heterotrophic conditions in the dark. When these $\mathrm{mt}^{-}$mutants were crossed with wild-type $\mathrm{mt}^{+}$cells, the germination rate was low (20-50\%) and the rate of paternal inheritance of mtDNA was about $85 \%$. In the reciprocal cross, this inheritance rate recovered.

Colin et al. (1995) isolated two additional mutants, dum-18 and dum-19, carrying frameshift mutations in coxl. They were unable to grow under heterotrophic conditions. These mutants showed the paternal inheritance of mitochondria.

Seven more mutants carrying a deletion or other mutation in the $c o b$ or coxl gene were isolated after treatment with acriflavine or ethidium bromide. These mutants were completely or almost completely unable to grow under heterotrophic (darkness + acetate) conditions (Dorthu et al. 1992). Paternal inheritance occurred when $\mathrm{mt}^{-}$wild-type and $\mathrm{mt}^{+}$mutant cells were crossed. However, upon reciprocal crossing, this paternal inheritance was disrupted.

Bennoun et al. (1991) isolated three mutants resistant to mucidin and myxothiazol. Diploid cells resistant to these inhibitors were first isolated and then crossed with wild-type haploid $\mathrm{mt}^{+}$cells for haploidization. Finally, the isolated mutant MUD2 showed strict paternal inheritance.

The above experiments raise questions about whether the process of paternal inheritance proceeds accurately in crosses between strains from different species (Boynton et al. 1987, Beckers et al. 1991), in crosses using respiration-deficient mutants (Matagne et al. 1989, Dorthu et al. 1992, Colin et al. 1995), or in haploid cells derived from artificially produced diploid cells (Bennoun et al. 1991).

\section{Paternal inheritance of mitochondrial DNA in C. reinhardtii}

To answer these questions, it is necessary to identify a suitable marker gene in the mitochondrial genome to determine parental origin. Nakamura et al. (2003) used the cob-1 intron gene as a marker. C. smithii mitochondria contain this gene, while $C$. reinhardtii mitochondria do not. These researchers successively performed interspecific backcrossing between strains of these two species in order to match their nuclear backgrounds and examined the inheritance of mtDNA at each backcross (Fig. 1). The mating efficiency increased from less than $1 \%$ to about $75 \%$ at the fourth step of backcrossing to $C$. reinhardtii. The frequency of complete tetrad production increased from less than $10 \%$ to almost $100 \%$ at the third step. An analysis of the progeny revealed that cpDNA was inherited maternally, while mtDNA was inherited strictly paternally.

\section{Period of disappearance of maternal mtDNA in zygotes}

Next, changes in the total amounts of mtDNA and cpDNA were tracked using real-time PCR and quantitative Southern blotting hybridization. As shown in Fig. 2, two periods, at about $6 \mathrm{~h}$ after zygote formation and just before the first meiotic division, were the candidates for the timing when maternal mtDNA disappears. To further analyze this issue, the fates of $\mathrm{mt}^{+}$and $\mathrm{mt}^{-}$parental coxl genes of mtDNA were followed. To obtain accurate data, a single zygote was picked up using a manipulator under a microscope and subjected to analysis. Between 6 and $12 \mathrm{~h}$ after the mature zygotes had been exposed to light, all $\mathrm{mt}^{+}$parental (female) mtDNA corresponfing to the lower band in the lane of $X b a$ I cut disappeared (Fig. 3). This time point was confirmed by FISH analysis (Fig. 4). The results show that the female mtDNA disappeared selectively between 6 and $18 \mathrm{~h}$ after exposure of the mature zygotes to light, that is, just before the first meiotic cell division.

\section{Gene expression at the early stage of germination}

When female and male gametes are mixed, they fuse to form diploid zygotes. During the first $24 \mathrm{~h}$ after fusion, the disappearance of flagella, selective elimination of paternal chloroplast DNA, nuclear membrane fusion, and chloroplast membrane fusion occur. The regulatory mechanisms involved in the maternal inheritance of cpDNA have been partially studied in terms of the transcription products of zygote-specific genes (Armbrust et al. 1993, Kuriyama et al. 1999, Uchida et al. 1999, Kubo et al. 2001). However, there are no reports on the transcription products at the early stage of germination of mature zygotes of $C$. reinhardtii.

Analysis using a next-generation sequencer (454 GS Junior) and quantitative real-time PCR identified genes expressed after exposure of mature zygotes to light (Aoyama et al. 2014). At 6 $\mathrm{h}$ of light exposure, the levels of expression of genes encoding antenna proteins involved in photosynthesis and two methionine metabolism-related genes were increased compared with those at $1 \mathrm{~h}$. Meanwhile, at $1 \mathrm{~h}$, the expression levels of three uncharacterized genes containing an antibiotic biosynthesis monooxygenase domain and an HSP20/alpha crystallin family protein increased. Further elucidation is necessary to reveal their involvement in mtDNA inheritance. 
A

B

Progeny backcrossed with C.s. (mt+) Progeny backcrossed with C.r. (mt-)

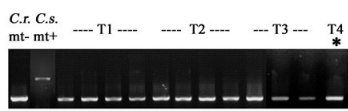

\section{$F_{1}$}

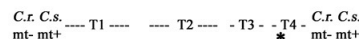

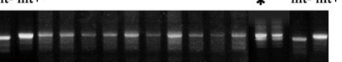

C.r. C.s.
$\mathrm{mt-m}-\mathrm{m}+\mathrm{T}$
$\mathbf{0}$

C.r. C.s.
mt-mt+
$\mathrm{mt}$

$\mathrm{F}_{2}$ emr-

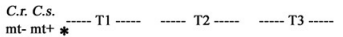

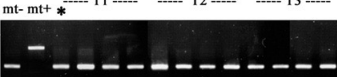

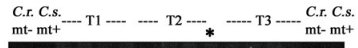

$\mathrm{F}_{3}$

C.r. C.s.
$\mathrm{mt}-\mathrm{mt+}$

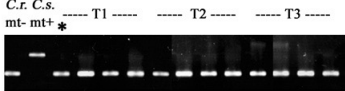

$\mathrm{F}_{4}$
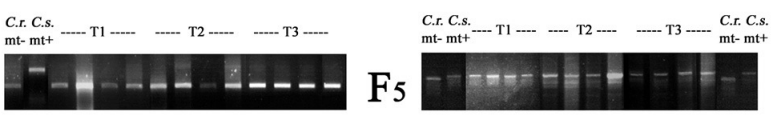

Figure 1 Inheritance of mitochondrial and chloroplast DNA during serial backcrosses. (A) Inheritance of mtDNA. (B) Inheritance of cpDNA. T1 to $\mathrm{T} 4$, tetrad numbers. Asterisk, progeny used for the following backcross. \#, exceptional paternal transmission. (reproduced from Nakamura et al. 2003)

A

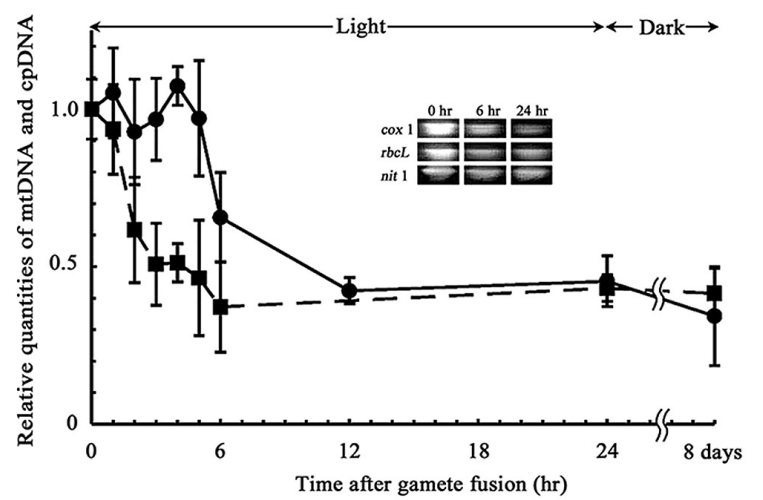

B

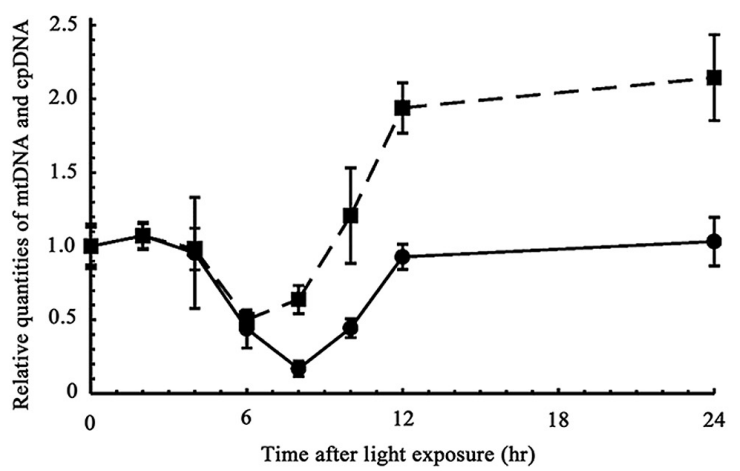

Figure 2 Changes in quantities of total mtDNA and total cpDNA relative to the quantity of nuclear DNA in zygotes. Quantities of mtDNA, cpDNA, and nuclear DNA were measured by real-time PCR. (A) Relative quantities of total mtDNA and total cpDNA at $24 \mathrm{~h}$ and eight days after gamete fusion. Inset, quantitative Southern blot hybridization of total mtDNA (coxl), total cpDNA ( $r b c L)$, and nuclear DNA (nit1). (B) Relative quantities of total mtDNA (solid line) and total cpDNA (dashed line) at $24 \mathrm{~h}$ after exposure of mature zygotes to light. (reproduced from Aoyama et al. 2006).

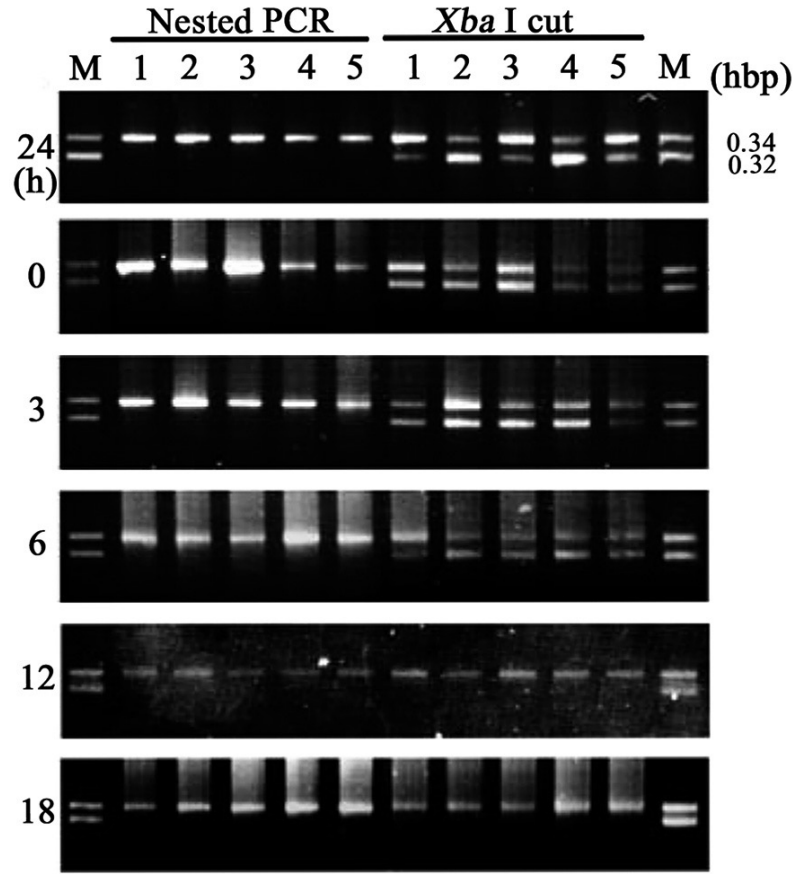

Figure 3 Disappearance of female mtDNA in zygotes. Nested PCR product of the coxl gene of isolated single zygotes was digested by the restriction enzyme $X b a \mathrm{I}$. Single zygotes were isolated $24 \mathrm{~h}$ after mixing of the two gametes and after exposure of mature zygotes to light. 1-5, number of single zygotes. $24 \mathrm{~h}, 24 \mathrm{~h}$ after gamete fusion. $0,3,6,12$, 18; Hours after exposure of mature zygotes to light. (reproduced from Aoyama et al. 2006).

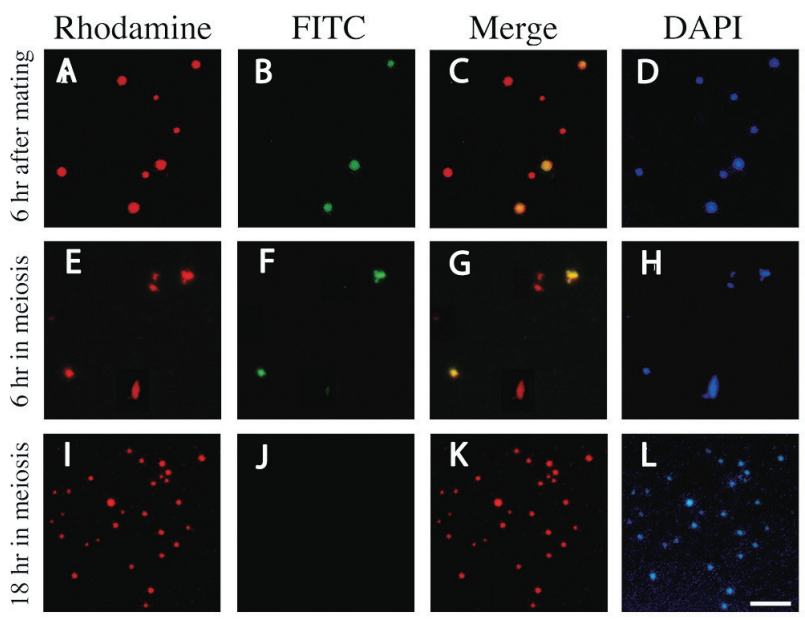

Figure 4 FISH analysis of the coxl and $c o b-1$ intron genes in mtDNA and DAPI staining of mitochondrial nucleoids. The $\mathrm{mt}^{-}$gamete (coxl gene) and the $\mathrm{mt}^{+}$gamete (coxl and cob-l intron genes) were fused. The rhodamine red signal indicates the coxl gene and the FITC green signal indicates the $c o b-1$ intron gene. (A-D) A zygote $6 \mathrm{~h}$ after gamete fusion. (E-H) A mature zygote $6 \mathrm{~h}$ after light exposure. (I-L) A mature zygote 18 $\mathrm{h}$ after light exposure. (A, E, I) Rhodamine signal from the coxl gene. (B, F, J) FITC signal from the cob- 1 intron gene. (C) Merged image of A and B. (G) Merged image of E and F. (K) Merged image of I and J. Overlap of the rhodamine (red) and FITC (green) signals produced a yellow color. (D) DAPI staining of the same cell used in A and B. (H) DAPI staining of the same cell used in E and F. (L) DAPI staining of the same cell used in I and J. Note that the FITC signal derived from $\mathrm{mt}^{+}$parental mtDNA disappeared between 6 and $18 \mathrm{~h}$ after light exposure. Bar $=5 \mu \mathrm{m}$. (reproduced from Aoyama et al. 2006). 
Mutant bp31 (biparental 31) with disruption of the maternal inheritance of cpDNA

Nishimura et al. (2012) isolated a mutant $b p 31$, having a deletion of about $60 \mathrm{~kb}$ on chromosome 2, in which the uniparental inheritance of cpDNA and mtDNA is disturbed (Fig. 5). This mutant phenotype was rescued by co-transformation with the GSP1 and inositol monophosphatase-like 1 genes (Table 1). This evidence shows that there is commonality between cpDNA and mtDNA in the biochemical process involved in the selective disappearance leading to uniparental inheritance.

\section{Observation of mitochondria and mitochondrial nucleoids of C. reinhardtii by fluorescence microscopy}

Ehara et al. (1995) observed mitochondria in C. reinhardtii cells by electron microscopy. They also observed these mitochondria by epifluorescence microscopy after staining with a mitochondrial membrane-binding fluorescent dye, dimethylaminostyrylmethylpyridiniumiodine. The mitochondria changed dynamically from granules to disk-shaped structures during the cell cycle. The morphology was classified into five types. The morphology of chloroplast nucleoids was also observed. The nucleoids were stained with DAPI after fixing with glutaraldehyde. The shapes of the chloroplast nucleoids changed from granular to thread-like, or in the opposite direction, but mitochondrial nucleoids were not observed.

In 1998, Nishimura et al. (1998) first stained cells with the DNA-specific fluorochrome SYBR Green I. They found that each cell contained about 60 mitochondrial granular nucleoids. SYBR Green I is better than DAPI for visualizing mitochondrial nucleoids because they are smaller than those of chloroplasts. Hiramatsu et al. (2006) stained the nucleoids of living cells cultured under a 12:12 light-dark cycle with SYBR Green I and the mitochondria with 3,3-dihexyloxacarbocyanine iodide 6 (DiOC 6 ) (Fig. 6). A mocG33 mutant containing one large chloroplast nucleoid throughout the cell cycle (Misumi et al. 1999, 2001) was used to distinguish between the mitochondrial

A

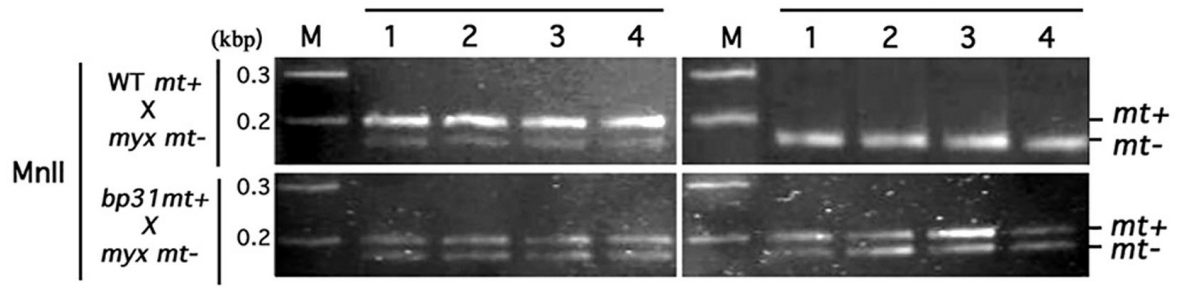

Figure 5 The $b p 31$ mutant is defective in paternal inheritance of mtDNA. Wild-type $\mathrm{mt}^{+}$and $b p 31 \mathrm{mt}^{+}$ gametes were crossed with myxothiazol-resistant gametes. Analysis was performed using nested PCR. (A) Cells at 30 min after fusion. (B) Fused cells incubated in the dark for one week and then in the light for 8 $\mathrm{h}$. Note that crossing with wild-type gametes showed only $\mathrm{mt}^{-} \mathrm{mtDNA}$, indicating paternal inheritance, but crossing with $b p 31$ showed $\mathrm{mt}^{+}$and $\mathrm{mt}^{-}$parental mtDNA, indicating biparental inheritance (Nishimura et al. 2012).

Table 1 Restored paternal inheritance of mtDNA in tetrad progeny. bp31+GSPlgu+INMIg (\#4, 13), Cotransformed strains of bp31 mutants with the two genes GSPI and INMI. MT, Mating type. mtDNA, Presence of the MnlI site, indicating paternal inheritance of mtDNA from the mt parent. (reproduced from Nishimura et al. 2012).

\begin{tabular}{|c|c|c|c|c|}
\hline Strain & Tetrad & Progeny & MT & mtDNA \\
\hline \multirow[t]{19}{*}{$\# 4$} & \multirow[t]{4}{*}{1} & 1 & - & mt- \\
\hline & & 2 & + & mt- \\
\hline & & 3 & - & mt- \\
\hline & & 4 & + & mt- \\
\hline & \multirow[t]{4}{*}{2} & 1 & - & mt- \\
\hline & & 2 & + & $\mathrm{mt}-$ \\
\hline & & 3 & - & mt- \\
\hline & & 4 & + & mt- \\
\hline & \multirow[t]{4}{*}{3} & 1 & - & mt- \\
\hline & & 2 & - & mt- \\
\hline & & 3 & + & mt- \\
\hline & & 4 & + & mt- \\
\hline & \multirow[t]{4}{*}{4} & 1 & + & mt- \\
\hline & & 2 & - & mt- \\
\hline & & 3 & + & mt- \\
\hline & & 4 & - & mt- \\
\hline & \multirow[t]{3}{*}{5} & 1 & + & mt- \\
\hline & & 2 & + & mt- \\
\hline & & 3 & - & $\mathrm{mt}-$ \\
\hline
\end{tabular}

bp31+ GSP1gu+ INM1g\#13 mt+ X myx mt-

\begin{tabular}{|c|c|c|c|c|}
\hline Strain & Tetrad & Progeny & MT & mtDNA \\
\hline \multirow[t]{17}{*}{$\# 13$} & \multirow[t]{4}{*}{1} & & + & mt- \\
\hline & & & + & mt- \\
\hline & & & - & mt- \\
\hline & & & - & mt- \\
\hline & \multirow[t]{2}{*}{2} & 1 & - & mt- \\
\hline & & 2 & - & mt- \\
\hline & \multirow[t]{2}{*}{3} & 1 & + & mt- \\
\hline & & 2 & - & mt- \\
\hline & \multirow[t]{3}{*}{4} & 1 & + & mt- \\
\hline & & 2 & + & mt- \\
\hline & & 3 & - & mt- \\
\hline & \multirow[t]{2}{*}{5} & 1 & + & mt- \\
\hline & & 2 & - & mt- \\
\hline & \multirow[t]{4}{*}{6} & 1 & - & mt- \\
\hline & & 2 & - & mt- \\
\hline & & 3 & + & mt- \\
\hline & & 4 & + & mt- \\
\hline
\end{tabular}


and chloroplast nucleoids (Fig. 7). At the start of the light period during the cell cycle, the new daughter cells contained about 30 mitochondrial and 10 chloroplast granular nucleoids, with diameters of 0.1 and $0.2 \mu \mathrm{m}$, respectively. They were dispersed across the entire cell. With time, both chloroplast and

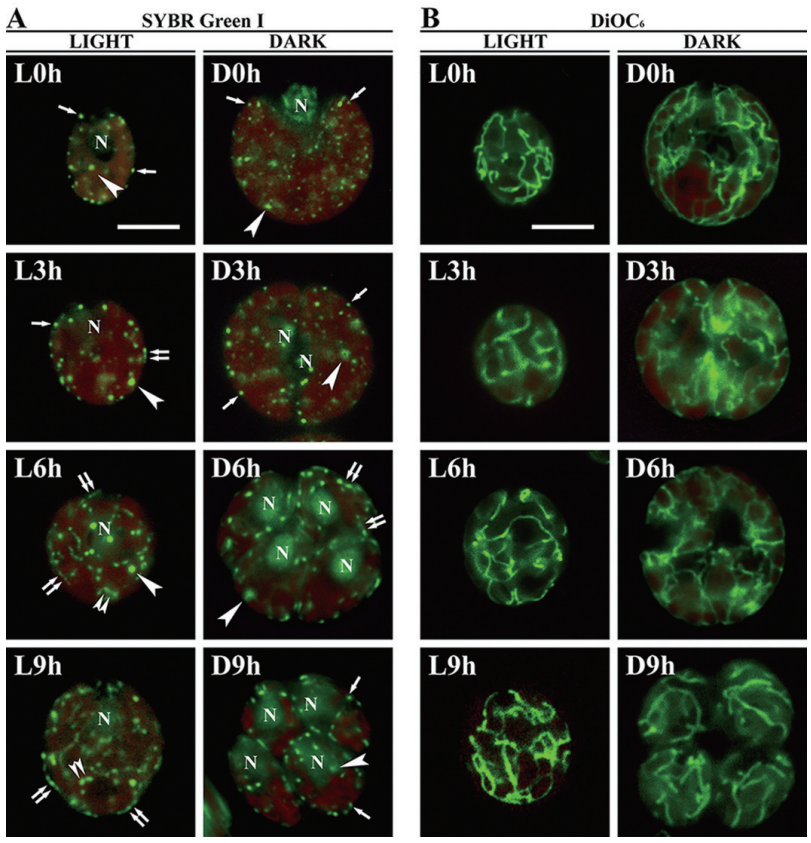

Figure 6 Fluorescence micrographs of wild-type cells stained with SYBR Green I (A) or $\mathrm{DiOC}_{6}$ (B). Cells were cultured under a 12:12 lightdark cycle. N, cell nucleus. Arrows, mitochondrial nucleoids. Double arrows, thread-like or bead-like structures of mt nucleoids. Arrowheads, chloroplast nucleoids. Double arrowheads, thread-like or bead-like structures of cp nucleoids. Bar $=5 \mu \mathrm{m}$. (reproduced from Hiramatsu et al. 2006).

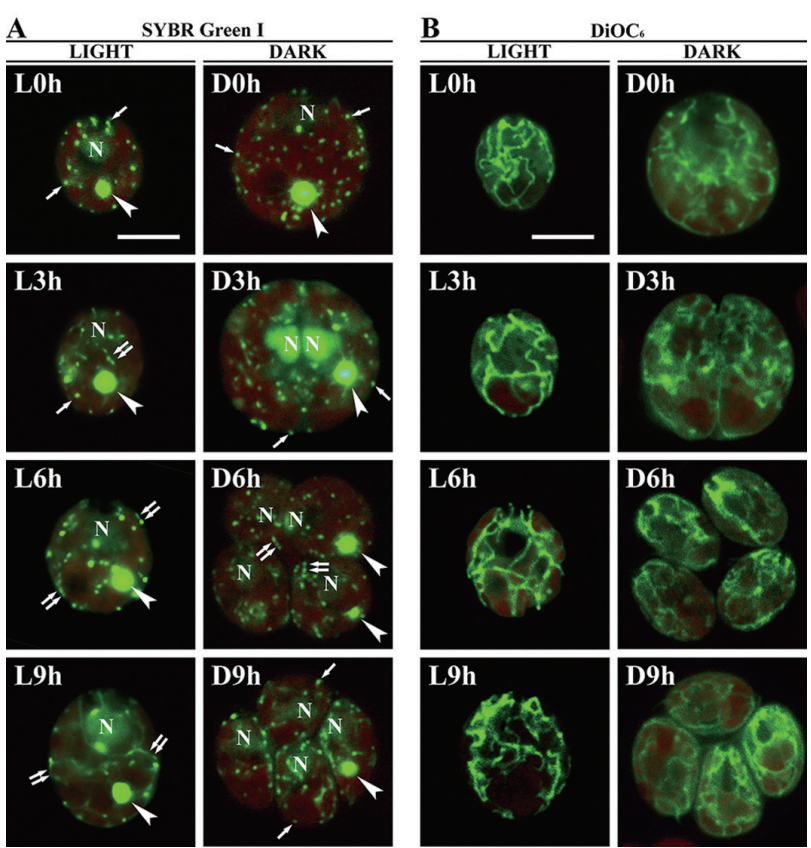

Figure 7 Fluorescence micrographs of mocG33 cells stained with SYBR Green I (A) or $\mathrm{DiOC}_{6}$ (B). Cells were cultured under a 12:12 light-dark cycle. N, cell nucleus. Arrows, mitochondrial nucleoids. Double arrows, bead-like structures of $\mathrm{mt}$ nucleoids. Arrowheads, chloroplast nucleoids. Bar $=5 \mu \mathrm{m}$. (reproduced from Hiramatsu et al. 2006). mitochondrial nucleoids continuously increased in number, but they then became smaller due to a decrease in the DNA content in each particle and became indistinguishable. They were usually granular, but some nucleoids formed short thread-like or beadlike structures, probably by dividing (double arrows in Fig. 7A). In the middle of the dark period, the nucleoids again formed short thread-like or bead-like structures, probably by fusing, and decreased in number. The mitochondria appeared as tangled sinuous structures that extended throughout the cytoplasm and resembled a single large mitochondrion.

Upon the maturation of zygotes, their walls became thick and solid, and they changed from green to dark black. Because of their thick walls, the zygote interior had been analyzed only using an electron microscope (Triemer \& Brown 1976, Storms \& Hastings 1977). Storms \& Hastings (1977) suggested that the haploid chromosome number is 18 or 20 . Before observing mitochondrial nucleoids in living cells, the chromosomes should be observed. Therefore, Aoyama et al. (2008) applied SYBR Green I staining to zygotes and counted a total of 18 chromosomes (Fig. 8).
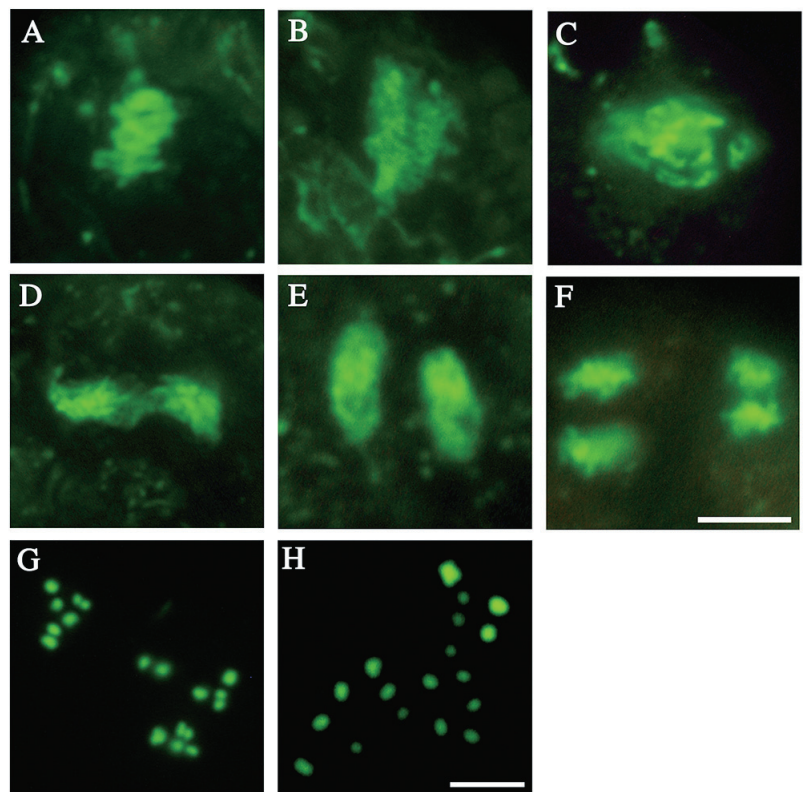

Figure 8 Chromosomes at metaphase and anaphase (A-E) and at meiosis II (F). Total chromosomes spread on a glass slide (G, H). Chromosomes were stained with SYBR Green I. (reproduced from Aoyama et al. 2008).

Aoyama et al. (2006, 2009) succeeded in visualizing mitochondrial nucleoids. They stained living mature zygotes with SYBR Green I for nucleoids and with $\mathrm{DiOC}_{6}$ for mitochondria and then traced them under an epifluorescence microscope after the exposure of mature zygotes to light (Fig. 9). Because discrimination between SYBR Green I-stained mitochondrial nucleoids and chloroplast nucleoids was impossible, as shown in Fig. 9, the zygotes were stained with SYBR Green I and $\mathrm{DiOC}_{6}$ simultaneously. Just after light exposure, the mitochondria were granular and scattered near the surface of the zygote (Fig. 9AF). At about $3 \mathrm{~h}$ after light exposure, the granular mitochondria had transformed into short, tubular structures (Fig. 9G, H). The 
tubular mitochondria contained mitochondrial nucleoids (Fig. 9I). Large plastid nucleoids were observed inside the zygotes and could be discriminated from mitochondrial nucleoids by their size. At about $7 \mathrm{~h}$, the tubular mitochondria formed branch-like structures and then scattered throughout the cell (Fig. 9M-R), which contained nucleoids (Fig. 9O). At about $11 \mathrm{~h}$, the scattered mitochondria and most mitochondrial nucleoids assembled around the cell nucleus. Once they had assembled there, the tubular mitochondria radiated into the other hemisphere through the surface layer at about $14 \mathrm{~h}$. The mitochondrial nucleoids were then distributed in the zygote. At about 16 and $20 \mathrm{~h}$, the first and second cell divisions produced four daughter cells. The mitochondria in these daughter cells were meshlike. Mitochondrial and chloroplast granular nucleoids were distributed throughout the whole zygote. The smaller nucleoids were mitochondrial and the larger ones were of chloroplasts.

Real-time PCR analysis of the changes in the total mtDNA amount, restriction enzyme digestion, and FISH analyses previously showed the significant disappearance of female mtDNA just before the first meiotic division (Aoyama et al. 2006). This period corresponds to Fig. 9M-X. Morphological changes leading to the preferential disappearance of $\mathrm{mt}^{+}$ mitochondrial nucleoids should be observed in this period. Because of the numerous tiny mitochondrial and plastid nucleoids, and the vigorous movements associated with the assembly and radiation of nucleoids just before meiosis, no significant disappearance of mitochondrial nucleoids was observed. Recently, Aoyama et al. (2015) observed mitochondria and their nucleoids by fluorescent double staining in living cells using a confocal fluorescence microscope and constructed a 3D model. Time lapse observation using this approach should provide definitive information on this issue. The above observations are summarized in Fig. 10.

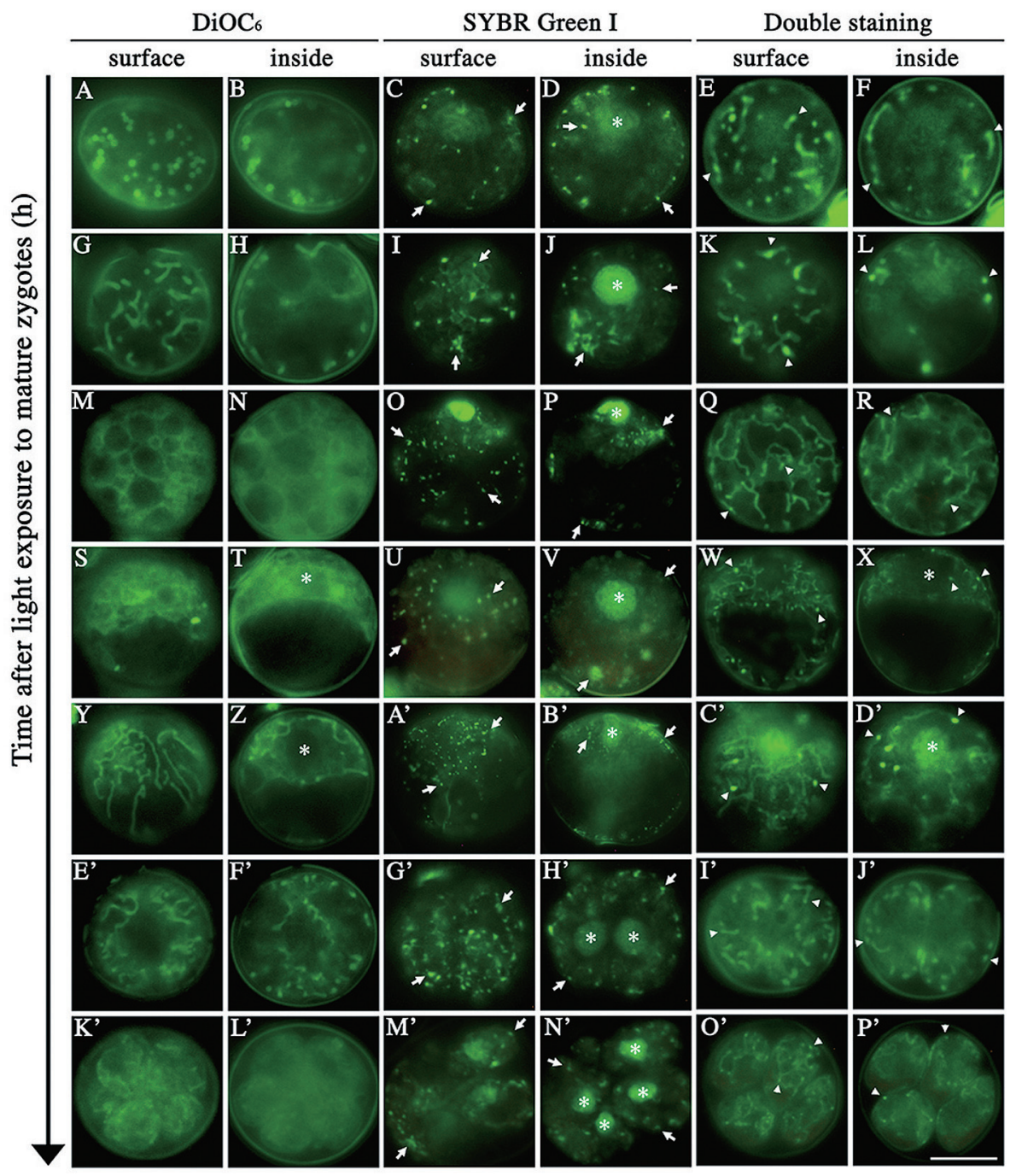

Figure 9 Fluorescence micrographs of mature zygotes after light exposure. Fluorescence micrographs of zygotes stained with $\mathrm{DiOC}_{6}$, SYBR Green I, and both SYBR Green I and $\mathrm{DiOC}_{6}$ (double staining) simultaneously. Surface, images focused on the surface layer of a single zygote. Inside, images focused on the inside of a single zygote. Asterisks, cell nucleus. Arrows, organellar nucleoids. Arrowheads, mitochondrial nucleoids. Bar $=5 \mu \mathrm{m}$. (reproduced from Aoyama et al. 2009). 

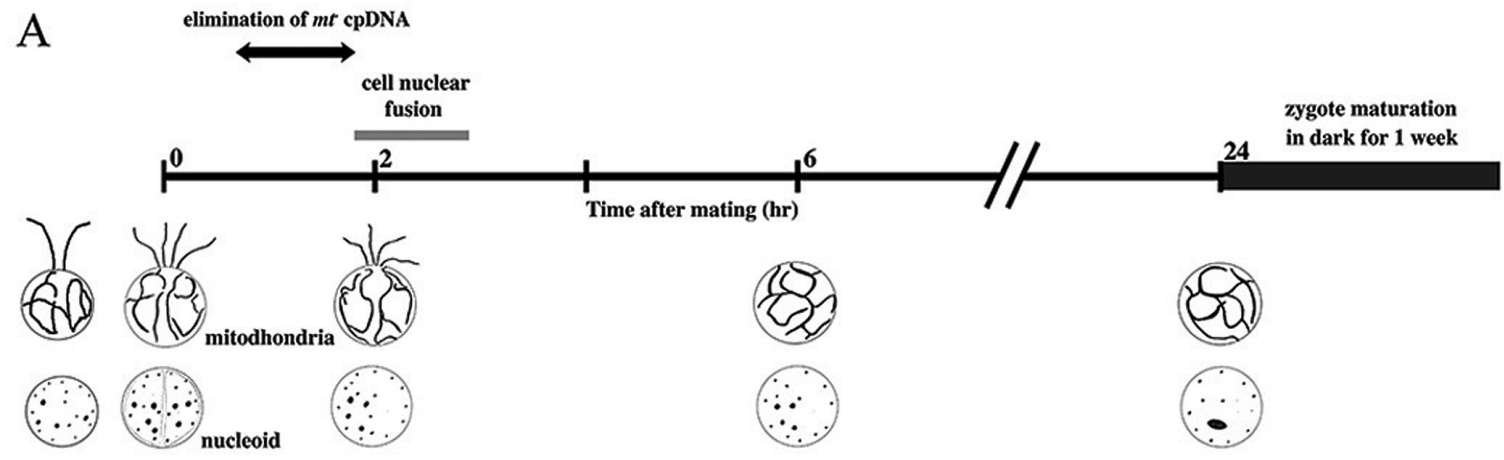

Gamete ZYgote

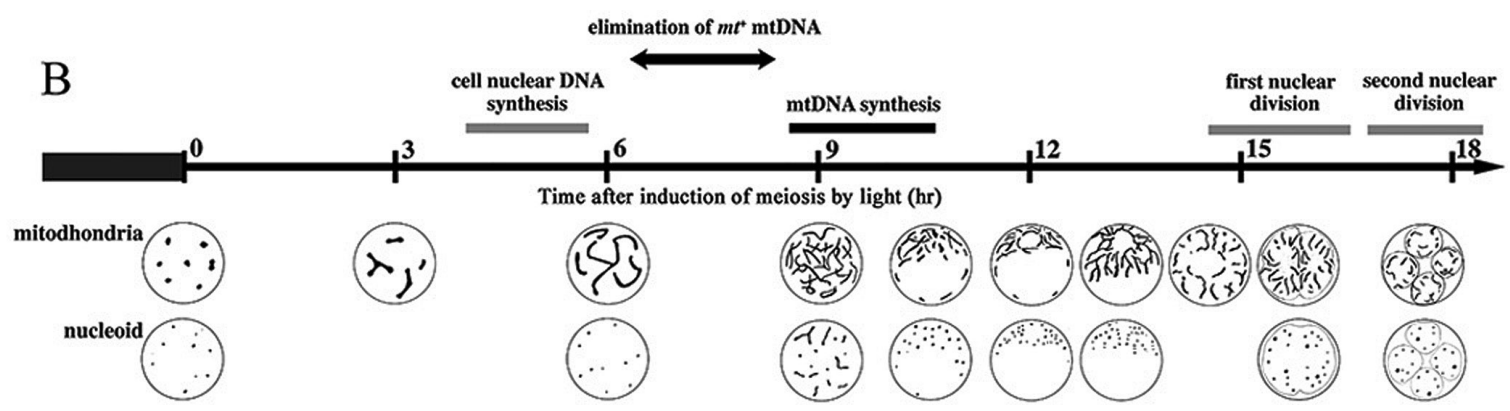

Figure 10 Timetable of the main events during the period between zygote formation and tetrad formation. (A) At about $1 \mathrm{~h}$, mt parental chloroplast nucleoids disappeared. At about $2 \mathrm{~h}$, the two cell nuclei fused. Mitochondrial nucleoids and mitochondria were scattered throughout the zygote. Numbers, hours after mixing male and female gametes. (B) When mature zygotes were exposed to light, $\mathrm{mt}^{+}$parental mtDNA selectively disappeared just before mtDNA synthesis. Some nucleoids subsequently began to form short thread-like structures. Mitochondria probably synthesize their DNA during this period, divide, and then increase in number. Then, the nucleoids become particles again. Once mitochondria and mitochondrial nucleoids had assembled around the cell nucleus, they dispersed radially to the other hemisphere. The first and second cell divisions subsequently occurred. Numbers, hours after exposure of mature zygotes to light. (reproduced from Aoyama et al. 2006).

\section{ACKNOWLEDGEMENTS}

I would like to thank Professor T. Kuroiwa for his generous support. I would also like to thank my coworkers Dr. H. Aoyama and Professor Y. Nishimura (Kyoto University), and other members of my laboratory.

\section{REFERENCES}

Armbrust, E. V., Ferris, P. J., and Goodenough, U. W. (1993) A mating type-linked gene cluster expressed in Chlamydomonas zygotes participates in the uniparental inheritance of the chloroplast genome. Cell 74: 801-811.

Aoyama, H., Hagiwara, Y., Misumi, O., Kuroiwa, T., and Nakamura, S. (2006) Complete elimination of maternal mitochondrial DNA during meiosis resulting in the paternal inheritance of the mitochondrial genome in Chlamydomonas species. Protoplasma 228: 231-242.

Aoyama, H., Kuroiwa, T., and Nakamura, S. (2008) Observations of chromosomal behaviour in living meiotic zygotes of Chlamydomonas reinhardtii (Chlorophyceae). Eur J Phycol 43: 389-394.

Aoyama, H., Kuroiwa, T., and Nakamura, S. (2009) The dynamic behaviour of mitochondria in living zygotes during maturation and meiosis in Chlamydomonas reinhardtii. Eur J Phycol 44: 497-507.

Aoyama, H., Ono, Y., Kuroiwa, T., and Nakamura, S. (2015) Live imaging of mitochondrial morphology during vegetative cell cycle in Chlamydomonas reinhardtii. Cytologia 80: 259-260.

Aoyama, H., Saitoh S., Kuroiwa T., and Nakamura S. (2014) Comparative analysis of zygospore transcripts during early germination in Chlamydomonas reinhardtii. J Plant Physiol 171: 1685-1692.

Baur, E. (1909) Das wesen und die erblichkeitsverhaltnisse der Varietates albo-marginatae hort von Pelargonium zonale. Z Vererbungsl 1: 330351 .

Beckers, M. C., Munaut, C., Minet, A., and Matagne, R. F. (1991) The fate of mitochondrial DNAs of $\mathrm{mt}^{+}$and $\mathrm{mt}^{-}$origin in gametes and zygotes of Chlamydomonas. Curr Genet 20: 239-243.

Bennoun, P., Delosme, M., and Kuckt, U. (1991) Mitochondrial genetics of Chlamydomonas reinhardtii: resistance mutations marking the cytochrome b gene. Genetics 127: 335-343.

Boynton, J. E., Harris, E. H., Burkhart, B. D., Lamerson, P. M., and Gillham, N. W. (1987) Transmission of mitochondrial and chloroplast genomes in crosses of Chlamydomonas. Proc Natl Acad Sci USA 84: 2391-2395.

Colin, M., Dorthu, M. P., Duby, F., Remacle, C., Dinant, M., Wolwertz, M. R., Duyckaerts, C., Sluse, F., and Matagne, R. F. (1995) Mutations affecting the mitochondrial genes encoding the cytochrome oxidase subunit I and apocytochrome b of Chlamydomonas reinhardtii. Mol Gen Genet 249: 179-184.

Correns, C. (1909) Vererbungsversuche mit blass (gelb) grunen und buntblattrigen Sippen bei Mirabilis jalapa, Urtica pilulifera und Lunaria annua. Z Vererbungsl 1: 291-329.

Dorthu, M. P., Remy, S., Michel-Wolwertz, M. R., Colleaux, L., Breyer, D., Beckers, M. C., Englebert, S., Duyckaerts, C., Sluse, F. E., and Matagne, R. F. (1992) Biochemical, genetic and molecular characterization of new respiratory- deficient mutants in Chlamydomonas reinhardtii. Plant Mol Biol 18: 759-772.

Ehara, T., Osafune, T., and Hase, E. (1995) Behavior of mitochondria in synchronized cells of Chlamydomonas reinhardtii (Chlorophyta). $J$ Cell Sci 108:499-507.

Gillham, N. W. (1994) Organelle genes and genomes. Oxford University Press, New York. 
Grant, D., and Chiang, K. S. (1980) Physical mapping and characterization of Chlamydomonas mitochondrial DNA molecules: their unique ends, sequence homogeneity, and conservation. Plasmid 4: 82-96.

Gyllensten U., Wharton D., Josefsson A., and Wilson A. C. (1991) Paternal inheritance of mitochondrial DNA in mice. Nature 352: 255-257.

Hiramatsu, T., Misumi, O., Kuroiwa, T., and Nakamura, S. (2006). Morphological changes in mitochondrial and chloroplast nucleoids and mitochondria during the Chlamydomonas reinhardtii (Chlorophyceae) cell cycle. J Phycol 42: 1048-1058.

Kubo, T., Abe, J., Oyamada, T., Ohnishi, M., Fukuzawa, H., Matsuda, Y., and Saito, T. (2008). Characterization of novel genes induced by sexual adhesion and gamete fusion and of their transcriptional regulation in Chlamydomonas reinhardtii. Plant Cell Physiol 49: 981-993.

Kuriyama, H., Takano, H., Suzuki, L., Uchida, H., Kawano, S., Kuroiwa, H., and Kuroiwa, T. (1999) Characterization of Chlamydomonas reinhardtii zygote-specific cDNAs that encode novel proteins containing ankyrin repeats and WW domains. Plant Physiol 119: 873-884.

Kuroiwa, T., Suzuki, T., Ogawa, K., and Kawano, S. (1981) The chloroplast nucleus: distribution, number, size, and shape, and a model for the multiplication of the chloroplast genome during chloroplast development. Plant Cell Physiol 22: 381-396.

Kuroiwa, T., Kawano, S., and Nishibayashi, S. (1982) Epifluorescent microscopic evidence for maternal inheritance of chloroplast DNA. Nature 298: 481-483.

Kuroiwa, T. (1985) Mechanisms of maternal inheritance of chloroplast DNA: an active digestion hypothesis. Microbiol Sci 2: 267-270.

Kuroiwa, T. (1991) The replication, differentiation and inheritance of plastids with emphasis on the concept of organelle nuclei. Int Rev Cytol 128: 1-62.

Kuroiwa, T. (2010) Review of cytological studies on cellular and molecular mechanisms of uniparental (maternal or paternal) inheritance of plastid and mitochondrial genomes induced by active digestion of organelle nuclei (nucleoids). J Plant Res 123: 207-230.

Liu, X. Q., Gillham, N. W., and Boynton, J. E. (1989) Chloroplast ribosomal protein gene rps12 of Chlamydomonas reinhardtii. Wildtype sequence, mutation to streptomycin resistance and dependence, and function in Escherichia coli. J Biol Chem 264: 16100-16108.

Matagne, R. F., Michel-Wolwertz, M. R., Munaut, C., Duyckaerts, C., and Sluse, F. (1989) Induction and characterization of mitochondrial DNA mutants in Chlamydomonas reinhardtii. J Cell Biol 108: 12211226.

Misumi, O., Suzuki, L., Nishimura, Y., Sakai, A., Kawano, S., Kuroiwa, H., and Kuroiwa, T. (1999) Isolation and phenotypic characterization of Chlamydomonas reinhardtii mutants defective in chloroplast DNA segregation. Protoplasma 209: 273-282.

Misumi, O., Nishimura, Y., and Kuroiwa, T. (2001) Effects of chloroplast DNA content on the cell proliferation and aging in Chlamydomonas reinhardtii. J Plant Res 14:125-131.

Nakamura, S., Aoyama, H., and Van-Woesik, R. (2003) Strict paternal transmission of mitochondrial DNA of Chlamydomonas species is explained by selection against maternal nucleoids. Protoplasma 221: 205-210.

Nishimura, Y., Higashiyama, T., Suzuki, L., Misumi, O., and Kuroiwa, T. (1998) The biparental transmission of the mitochondrial genome in Chlamydomonas reinhardtii visualized in living cells. Eur J Cell Biol 77: 124-133.

Nishimura, Y., Shikanai, T., Nakamura, S., Kawai-Yamada, M., and Uchiyama, H. (2012) Gspl triggers the sexual developmental program including inheritance of chloroplast DNA and mitochondrial DNA in Chlamydomonas reinhardtii. Plant Cell 24: 2401-2414.

Ris, H. and Plaut, W. (1962) Ultrastructure of DNA-containing areas in the chloroplast of Chlamydomonas. J Cell Biol 13: 383-391.

Ryan, R., Grant, D., Chiang, K.S., and Swift, H. (1978) Isolation and characterization of mitochondrial DNA from Chlamydomonas reinhardtii. Proc Natl Acad Sci USA 75: 3268-3272.

Sager, R. (1954) Mendelian and non-Mendelian inheritance of streptomycin resistance in Chlamydomonas reinhardi. Proc Natl Acad Sci USA 40: 356-363.

Sager, R. and Ishida, M. R. (1963) Chloroplast DNA in Chlamydomonas. Proc Natl Acad Sci USA 50:725-730.

Sager, R. and Lane, D. (1972) Molecular basis of maternal inheritance. Proc Natl Acad Sci USA 69: 2410-2413.

Storms, R. and Hastings, P. J. (1977) A fine structure analysis of meiotic pairing in Chlamydomonas reinhardii. Exp Cell Res 104: 39-46.

Sutton, W. S. (1902) On the morphology of the chromosome group in Brachystola magna. Biol Bull 4: 24-39.

Tilney-Bassett, R. A. E. (1978) The inheritance and genetic behaviour of plastids. In: Kirk, J. T. O. and Tilney-Bassett, R. A. E. (eds) The plastids: their chemistry, structure, growth and inheritance. Elsevier/ North-Holland Biomedical, New York, pp. 251-521.

Triemer, R. E. and Brown, R. M. (1976) Ultrastructure of meiosis in Chlamydomonas reinhardtii. Br Phycol J 12: 23-44.

Uchida, H., Suzuki, L., Anai, T., Doi, K., Takano, H., Yamashita, H., Oka, T., Kawano, S., Tomizawa, K.I., Kawazu, T., Kuroiwa, H., and Kuroiwa, T. (1999) A pair of invertedly repeated genes in Chlamydomonas reinhardtii encodes a zygote-specific protein whose expression is UV-sensitive. Curr Genet 36: 232-240.

Received: 16 December 2015 / Accepted: 20 January 2016 